\title{
Robust Exponential Stability of Impulsive Stochastic Neural Networks with Leakage Time-Varying Delay
}

\author{
Chunge Lu and Linshan Wang \\ College of Mathematical Science, Ocean University of China, Qingdao 266100, China \\ Correspondence should be addressed to Chunge Lu; chglu@126.com
}

Received 26 March 2014; Revised 3 July 2014; Accepted 9 July 2014; Published 24 July 2014

Academic Editor: Ademir F. Pazoto

Copyright (C) 2014 C. Lu and L. Wang. This is an open access article distributed under the Creative Commons Attribution License, which permits unrestricted use, distribution, and reproduction in any medium, provided the original work is properly cited.

\begin{abstract}
This paper investigates mean-square robust exponential stability of the equilibrium point of stochastic neural networks with leakage time-varying delays and impulsive perturbations. By using Lyapunov functions and Razumikhin techniques, some easy-to-test criteria of the stability are derived. Two examples are provided to illustrate the efficiency of the results.
\end{abstract}

\section{Introduction}

In recent years, stability for neural networks with time delay has been extensively studied due to their great applications in some practical engineering problems such as signal processing, associate memory, and combinatorial optimization (see [1-3]). In particular, the leakage delay, which exists in the negative feedback terms (known as forgetting or leakage terms) of the system, has great impact on the dynamical behavior of neural networks (see [4-10]). Gopalsamy [4] initially discussed the problem of bidirectional associative memory neural networks with constant delays in the leakage term by using model transformation technique. Then, many results of stability of neural networks with delays in the leakage terms are obtained (see, [5-12]).

However, besides time delay, neural networks are often subject to impulsive perturbation-the abrupt changes at certain instants, which may be caused by switching phenomenon, frequency change, or other sudden noise (see [13, 14]). The impulsive effect can affect the dynamical behaviors of the system. Now there are many results on stability of the neural networks with time delays in the leakage term and impulsive perturbations under the corresponding delayed neural networks without impulses must be stable themselves (see [5-8]). To best of the authors' knowledge, this is the first attempt to investigate the stability of the systems under the corresponding delayed neural networks without impulses which are unstable themselves.

On the other hand, in real nervous systems, the synaptic transmission is a noisy process brought on by random fluctuations from the release of neurotransmitters and other probabilistic causes [15]. It is well known that a neural network could be stabilized or destabilized by certain stochastic inputs. Therefore, noise disturbances have an important effect on the stability of neural networks. Recently, many interesting results on stochastic effect to the stability of neural networks with delays have been reported (see [11, $16,17])$. Moreover, uncertainties are unavoidable in practical implementation of neural networks due to modeling errors and parameter fluctuation, which also cause instability and poor performance [12]. Hence, we can obtain a more perfect model of this situation if we include parameter uncertainties and stochastic effects in neural networks.

Motivated by the above, it is of practical and theoretical importance to study the stability problem of impulsive neural networks with time-varying delays in the leakage term. In this paper, we will investigate stability for a class of stochastic neural networks with time-varying delay in the leakage term and impulses. By using Razumikhin techniques [18-21], some new robust mean-square exponential stability criteria will be given under the corresponding delayed neural networks without impulses which are stable and unstable, respectively. 


\section{Problem Formulation}

Consider the following uncertain neural networks model with impulses and leakage time-varying delay

$$
\begin{aligned}
d x(t)=[ & -C(t) x(t-\sigma(t))+A(t) f(x(t)) \\
& +B(t) f(x(t-\tau(t)))] d t \\
+ & h(x(t), x(t-\tau(t)), x(t-\sigma(t))) d \omega(t), \\
& t \geq t_{0}, \quad t \neq t_{k} ; \\
\Delta x(t)= & I_{k}\left(x\left(t^{-}\right), x\left(t^{-}-\tau(t)\right), x\left(t^{-}-\sigma(t)\right)\right), \\
& t=t_{k}, \quad k \in \mathbb{N}, \\
& x\left(t_{0}+s\right)=\varphi(s), \quad s \in[-\rho, 0],
\end{aligned}
$$

where $x(t)=\left(x_{1}(t), x_{2}(t), \ldots, x_{n}(t)\right)^{T} \in \mathbb{R}^{n}$ is the neuron state vector of the neural networks, $C(t)=\operatorname{diag}\left(c_{i}(t)\right)_{n \times n}>0$, $f(x)=\left(f_{1}\left(x_{1}\right), f_{2}\left(x_{2}\right), \ldots, f_{n}\left(x_{n}\right)\right)^{T}$ represents the neuron activation function, and $A(t), B(t) \in \mathbb{R}^{n \times n}$ are the connection weight matrix. $\tau(t)$ is the time-varying delay and $\sigma(t)$ is the leakage time-varying delay satisfying $0 \leq \tau(t) \leq \tau, 0 \leq$ $\sigma(t) \leq \sigma, \rho=\max (\tau, \sigma) . \omega(t)=\left(\omega_{1}(t), \omega_{2}(t), \ldots, \omega_{n}(t)\right)^{T}$ is a $n$-dimensional Brownian motion defined on complete probability space $(\Omega, \mathscr{F}, \mathscr{P}) . t_{k} \in J=\left\{\left\{t_{k}\right\}: 0=t_{0}<\right.$ $\left.t_{1}<\cdots<t_{k}<\cdots, \lim _{k \rightarrow \infty} t_{k} \rightarrow \infty\right\}$ is impulsive time. $\Delta x(t)=x\left(t_{k}\right)-x\left(t_{k}^{-}\right)$represents the jump in the state $x$ at $t_{k}$ with $I_{k}$ determining the size of the jump and $x\left(t_{k}^{+}\right)=$ $x\left(t_{k}\right)$. The initial conditions $\varphi(t) \in P C_{\mathscr{F}_{0}}^{b}\left([-\rho, 0] ; \mathbb{R}^{n}\right)$, where $P C_{\mathscr{F}_{0}}^{b}\left([-\rho, 0] ; \mathbb{R}^{n}\right)$ denotes the family of all bounded $\mathscr{F}_{0}$ measurable and $P C\left([-\rho, 0], \mathbb{R}^{n}\right)$ valued random variable $\varphi$, satisfying $\mathbb{E}\|\varphi\|^{2}=\sup _{-\rho \leq s \leq 0} \mathbb{E}|\varphi(s)|^{2}<+\infty ; \mathbb{E}$ denotes the mathematical expectation. $P C\left(J, \mathbb{R}^{n}\right)=\{\varphi: J \rightarrow$ $\mathbb{R}^{n}$ is piecewise continuous\}.

Throughout this paper, symmetric matrix $M \geq 0$ (resp., $M>0$ ) means that the matrix $M$ is positive semidefinite (resp., positive definite). I denotes an identity matrix. The notation $M^{T}$ represents the transpose of the matrix $M$. The symmetric terms in asymmetric matrix are denoted by $*$. $\lambda_{\text {max }}(A)$ and $\lambda_{\text {min }}(A)$ mean the largest and the smallest eigenvalue of $A$, respectively.

In this paper, we assume that $x^{*}=\left(x_{1}^{*}, x_{2}^{*}, \ldots, x_{n}^{*}\right)^{T}$ is the equilibrium point of the system (1). And we have the following assumptions.

$\left(\mathrm{A}_{1}\right)$ The neuron activation function $f_{j}(x)$ is continuous on $\mathbb{R}$ and satisfies

$$
l_{j} \leq \frac{f_{j}(u)-f_{j}(v)}{u-v} \leq L_{j},
$$

$$
j=1,2, \ldots, n, \quad \text { for any } u, v \in \mathbb{R}, \quad u \neq v,
$$

where $l_{j}, L_{j}$ are some real constants and they may be positive, zero, or negative.
$\left(A_{2}\right)$ Consider

$$
\begin{aligned}
I_{k}(x & \left.\left(t^{-}\right), x\left(t^{-}-\tau(t)\right), x\left(t^{-}-\sigma(t)\right)\right) \\
= & \left(G_{1 k}(t)-I\right)\left(x\left(t^{-}\right)-x^{*}\right)+G_{2 k}(t)\left(x\left(t^{-}-\tau(t)\right)-x^{*}\right) \\
& +G_{3 k}(t)\left(x\left(t^{-}-\sigma(t)\right)-x^{*}\right),
\end{aligned}
$$

where $G_{i k}(t) \in \mathbb{R}^{n \times n}, i=1,2,3$, and $k \in \mathbb{N}$.

$\left(\mathrm{A}_{3}\right)$ Consider $h(x(t), x(t-\tau(t)), x(t-\sigma(t)))=H_{1}(t)(x(t)-$ $\left.x^{*}\right)+H_{2}(t)\left(x(t-\tau(t))-x^{*}\right)+H_{3}(t)\left(x(t-\sigma(t))-x^{*}\right)$. Let $y(t)=x(t)-x^{*}$, and system (1) becomes

$$
\begin{array}{r}
d y(t)=[-C(t) y(t-\sigma(t))+A(t) g(y(t)) \\
+B(t) g(y(t-\tau(t)))] d t \\
+\left[H_{1}(t) y(t)+H_{2}(t) y(t-\tau(t))\right. \\
\left.+H_{3}(t) y(t-\sigma(t))\right] d \omega(t), \\
t \geq t_{0}, \quad t \neq t_{k} ;
\end{array}
$$

$$
\begin{aligned}
\Delta y(t)= & \left(G_{1 k}(t)-I\right) y\left(t^{-}\right)+G_{2 k}(t) y\left((t-\tau(t))^{-}\right) \\
& +G_{3 k}(t) y\left((t-\sigma(t))^{-}\right), \quad t=t_{k}, k \in \mathbb{N}, \\
& y\left(t_{0}+s\right)=\phi(s), \quad s \in[-\rho, 0],
\end{aligned}
$$

where $g(y(t))=f(x(t))-f\left(x^{*}\right)=$ $\left(g_{1}\left(y_{1}(t)\right), g_{2}\left(y_{2}(t)\right), \ldots, g_{n}\left(y_{n}(t)\right)\right)^{T}, \phi(s)=\varphi(s)-x^{*}$.

$\left(\mathrm{A}_{4}\right)$ We consider the parameter uncertainties expressed as

$$
\begin{aligned}
& A(t)=A+\Delta A(t), \quad B(t)=B+\Delta B(t), \\
& C(t)=C+\Delta C(t), \\
& H_{i}(t)=H_{i}+\Delta H_{i}(t), \quad G_{i k}(t)=G_{i k}+\Delta G_{i k}(t), \\
& i=1,2,3, \quad k \in \mathbb{N},
\end{aligned}
$$

where $A, B, C, H_{i}$, and $G_{i k}$ are known real constant matrices; $\Delta A(t), \Delta B(t), \Delta C(t), \Delta H_{i}(t)$, and $\Delta G_{i k}(t)$ are unknown matrices representing the parameter uncertainties, which are assumed to be the following form:

$[\Delta C(t) \Delta A(t) \Delta B(t)]=E_{1} F_{1}(t)\left[\begin{array}{lll}M_{1} & M_{2} & M_{3}\end{array}\right]$,

$\left[\begin{array}{lll}\Delta H_{1}(t) & \Delta H_{2}(t) & \Delta H_{3}(t)\end{array}\right]=E_{2} F_{2}(t)\left[\begin{array}{lll}N_{1} & N_{2} & N_{3}\end{array}\right]$,

$\left[\begin{array}{lll}\Delta G_{1 k}(t) & \Delta G_{2 k}(t) & \Delta G_{3 k}(t)\end{array}\right]=E_{3} F_{2}(t)\left[\begin{array}{lll}U_{1} & U_{2} & U_{3}\end{array}\right]$,

where $U_{i}, E_{i}, M_{i}, N_{i}(i=1,2,3)$ are known real constant matrices and $F_{i}(t)$ are unknown real timevarying matrix functions satisfying $F_{i}^{T}(t) F_{i}(t) \leq I$, $i=1,2,3$.

Remark 1. Assumptions $\left(\mathrm{A}_{1}\right)-\left(\mathrm{A}_{4}\right)$ imply that system (4) satisfies the local Lipschitz condition and linear growth condition. Thus there exists a unique solution of system (4). 
Definition 2. The equilibrium point $x^{*}$ of system (1) is said to be robustly exponentially stable in the mean square, if there exists scalars $\gamma>0$ and $\delta>0$ such that for any $\varepsilon>0$ and initial condition $\varphi$ satisfying $\mathbb{E}\|\varphi\| \leq \delta$ which implies $\mathbb{E}\left|x\left(t ; t_{0}, \varphi\right)-x^{*}\right|<\varepsilon e^{-\gamma\left(t-t_{0}\right)}, t \geq t_{0}$.

Lemma 3 (see [22]). Given matrices $D, E$, and $F$ with $F^{T} F \leq I$ and a scalar $\varepsilon>0$, then

$$
D F E+E^{T} F^{T} D^{T} \leq \varepsilon D D^{T}+\varepsilon^{-1} E^{T} E .
$$

\section{Main Results}

Theorem 4. Suppose that assumptions $\left(A_{1}\right)-\left(A_{4}\right)$ hold and for prescribed scalar $\Delta_{\max }>0$, choose positive scalars $\alpha, \beta$, and $\mu_{1}>0, \mu_{2}>0, \mu_{3}>0$, such that $\mu_{1}+\mu_{2}+\mu_{3}<1$. Then the equilibrium point of system (1) is robustly exponentially stable in the mean square over any impulse time sequences satisfying $\sup \left\{t_{k}-t_{k-1} \mid k=1,2, \ldots\right\} \leq \Delta_{\max }$, if there exist positive definite matrix $P>0$ and definite diagonal matrices $W_{1}, W_{2}$, and positive constants $\varepsilon_{1}, \varepsilon_{2}, \varepsilon_{3}$ such that the following LMI holds:

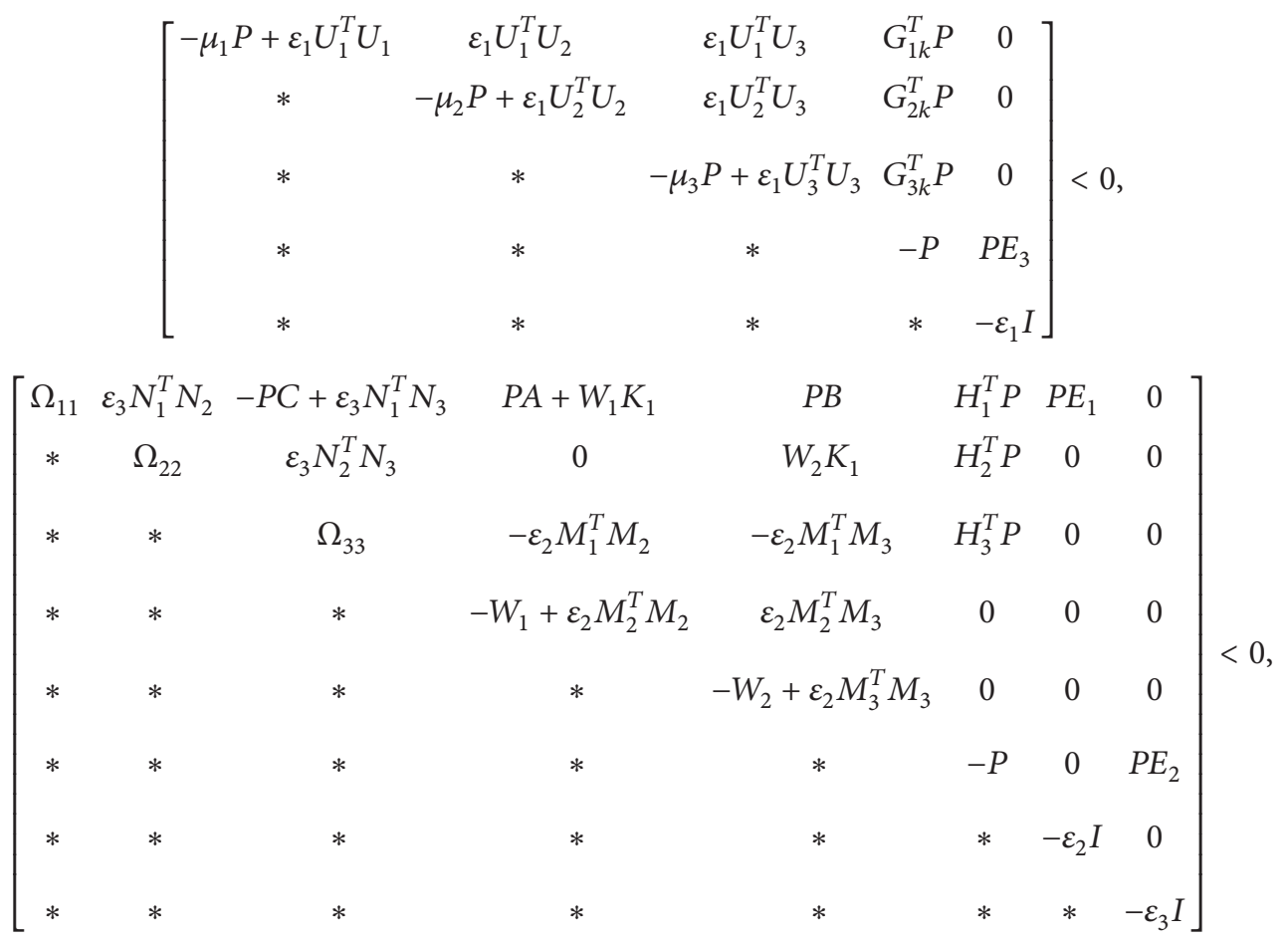

where $\Omega_{11}=\left[(\alpha+\beta) /\left(\mu_{1}+\mu_{2}+\mu_{3}\right)+\ln \left(\mu_{1}+\mu_{2}+\mu_{3}\right) / \Delta_{\text {max }}\right] P-$ $W_{1} K_{2}+\varepsilon_{3} N_{1}^{T} N_{1}, \Omega_{22}=-\alpha P-W_{2} K_{2}+\varepsilon_{3} N_{2}^{T} N_{2}, \Omega_{33}=$ $-\beta P+\varepsilon_{2} M_{1}^{T} M_{1}+\varepsilon_{3} N_{3}^{T} N_{3}, K_{1}=\operatorname{diag}\left(\left(\left(l_{1}+L_{1}\right) / 2\right),\left(\left(l_{2}+\right.\right.\right.$ $\left.\left.\left.L_{2}\right) / 2\right), \ldots,\left(\left(l_{n}+L_{n}\right) / 2\right)\right), K_{2}=\operatorname{diag}\left(l_{1} L_{1}, l_{2} L_{2}, \ldots, l_{n} L_{n}\right)$.
Proof. Since the matrix inequality (9) holds, we can choose small enough scalars $\eta>0, h>0$ satisfying $\mu_{1}+\mu_{2} e^{2 \eta \tau}+$ $\mu_{3} e^{2 \eta \sigma}<1$ and $h<1-\mu_{1}-\mu_{2} e^{2 \eta \tau}-\mu_{3} e^{2 \eta \sigma}$, such that

$$
\left[\begin{array}{cccccccc}
\bar{\Omega}_{11} & \varepsilon_{3} N_{1}^{T} N_{2} & -P C+\varepsilon_{3} N_{1}^{T} N_{3} & P A+W_{1} K_{1} & P B & H_{1}^{T} P & P E_{1} & 0 \\
* & \bar{\Omega}_{22} & \varepsilon_{3} N_{2}^{T} N_{3} & 0 & W_{2} K_{1} & H_{2}^{T} P & 0 & 0 \\
* & * & \bar{\Omega}_{33} & -\varepsilon_{2} M_{1}^{T} M_{2} & -\varepsilon_{2} M_{1}^{T} M_{3} & H_{3}^{T} P & 0 & 0 \\
* & * & * & -W_{1}+\varepsilon_{2} M_{2}^{T} M_{2} & \varepsilon_{2} M_{2}^{T} M_{3} & 0 & 0 & 0 \\
* & * & * & * & -W_{2}+\varepsilon_{2} M_{3}^{T} M_{3} & 0 & 0 & 0 \\
* & * & * & * & -P & 0 & P E_{2} \\
* & * & * & * & * & * & -\varepsilon_{2} I & 0 \\
* & * & * & * & * & * & * & -\varepsilon_{3} I
\end{array}\right]<0,
$$


where $\bar{\Omega}_{11}=\left[2 \eta+(\alpha+\beta) /\left(\mu_{1}+\mu_{2} e^{2 \eta \tau}+\mu_{3} e^{2 \eta \sigma}\right)+\ln \left(\mu_{1}+\mu_{2} e^{2 \eta \tau}+\right.\right.$ $\left.\left.\mu_{3} e^{2 \eta \sigma}+h\right) / \Delta_{\max }\right] P-W_{1} K_{2}+\varepsilon_{3} N_{1}^{T} N_{1}, \bar{\Omega}_{22}=-\alpha e^{-2 \eta \tau} P-$ $W_{2} K_{2}+\varepsilon_{3} N_{2}^{T} N_{2}, \bar{\Omega}_{33}=-\beta e^{-2 \eta \sigma} P+\varepsilon_{2} M_{1}^{T} M_{1}+\varepsilon_{3} N_{3}^{T} N_{3}$.

Let $q=1 /\left(\mu_{1}+\mu_{2} e^{2 \eta \tau}+\mu_{3} e^{2 \eta \sigma}\right), \lambda=-\ln \left(\mu_{1}+\mu_{2} e^{2 \eta \tau}+\right.$ $\left.\mu_{3} e^{2 \eta \sigma}+h\right) / \Delta_{\max }$; then $\lambda>0, q>1, e^{\lambda \Delta_{\max }}<q$.

Define Lyapunov function

$$
V(t, y(t))=e^{2 \eta t} y^{T}(t) P y(t) .
$$

From the condition (8), applying Schur complement [23] and Lemma 3, we have

$$
\begin{aligned}
& {\left[\begin{array}{ccc}
-\mu_{1} P & 0 & 0 \\
0 & -\mu_{2} P & 0 \\
0 & 0 & -\mu_{3} P
\end{array}\right]+\left[\begin{array}{c}
G_{1 k}^{T}(t) \\
G_{2 k}^{T}(t) \\
G_{3 k}^{T}(t)
\end{array}\right]} \\
& \quad \times P\left[G_{1 k}(t) \quad G_{2 k}(t) \quad G_{3 k}(t)\right]<0 .
\end{aligned}
$$

Therefore, when $t=t_{k}$,

$$
\begin{aligned}
V\left(t_{k}\right)= & e^{2 \eta t} y^{T}\left(t_{k}\right) P y\left(t_{k}\right) \\
= & e^{2 \eta t}\left[\begin{array}{c}
y\left(t_{k}^{-}\right) \\
y\left(t_{k}^{-}-\tau\left(t_{k}\right)\right) \\
y\left(t_{k}^{-}-\sigma\left(t_{k}\right)\right)
\end{array}\right]^{T}\left[\begin{array}{c}
G_{1 k}^{T}(t) \\
G_{2 k}^{T}(t) \\
G_{3 k}^{T}(t)
\end{array}\right] \\
& \left.\times P\left[\begin{array}{lll}
G_{1 k}(t) & G_{2 k}(t) & \left.G_{3 k}(t)\right]
\end{array}\right] \begin{array}{c}
y\left(t_{k}^{-}\right) \\
y\left(t_{k}^{-}-\tau\left(t_{k}\right)\right) \\
y\left(t_{k}^{-}-\sigma\left(t_{k}\right)\right)
\end{array}\right] \\
\leq & \mu_{1} V\left(t_{k}^{-}\right)+\mu_{2} e^{2 \eta \tau} V\left(t_{k}^{-}-\tau\left(t_{k}\right)\right) \\
& +\mu_{3} e^{2 \eta \sigma} V\left(t_{k}^{-}-\sigma\left(t_{k}\right)\right) .
\end{aligned}
$$

When $t \neq t_{k}$, applying the Itô formula, we have

$$
\begin{gathered}
\mathscr{L} V(t, y(t))=2 \eta e^{2 \eta t} y^{T}(t) P y(t)+2 e^{2 \eta t} y^{T}(t) \\
\times P[-C(t) y(t-\sigma(t))+A(t) g(y(t)) \\
+B(t) g(y(t-\tau(t)))] \\
+e^{2 \eta t}\left[H_{1}(t) y(t)+H_{2}(t) y(t-\tau(t))\right. \\
\left.+H_{3}(t) y(t-\sigma(t))\right]^{T} \\
\times P\left[H_{1}(t) y(t)+H_{2}(t) y(t-\tau(t))\right. \\
\left.+H_{3}(t) y(t-\sigma(t))\right] .
\end{gathered}
$$

Let

$$
\begin{aligned}
W(t)= & \mathscr{L} V(t)+\alpha(q V(t)-V(t-\tau(t))) \\
& +\beta(q V(t)-V(t-\sigma(t)))-\lambda V(t) .
\end{aligned}
$$

From assumption $\left(A_{1}\right)$, the following inequalities hold for any diagonal matrices $W_{1}>0, W_{2}>0$,

$$
\begin{aligned}
& e^{2 \eta t}\left[g(y(t)) W_{1} g(y(t))-2 y^{T}(t) W_{1} K_{1} g(y(t))\right. \\
& \left.+y^{T}(t) W_{1} K_{2} y(t)\right] \leq 0, \\
& e^{2 \eta t}\left[g(y(t-\tau(t))) W_{2} g(y(t-\tau(t)))-2 y^{T}(t-\tau(t))\right. \\
& \quad \times W_{2} K_{1} g(y(t-\tau(t)))+y^{T}(t-\tau(t)) \\
& \left.\quad \times W_{2} K_{2} y(t-\tau(t))\right] \leq 0 .
\end{aligned}
$$

Set

$\xi^{T}(t)=\left(y^{T}(t), y^{T}(t-\tau(t)), y^{T}(t-\sigma(t)), g^{T}(y(t))\right.$,

$$
\left.g^{T}(y(t-\tau(t)))\right) .
$$

Combining (15)-(16) together, we have

$$
W(t) \leq e^{2 \eta t} \xi^{T}(t) \Psi \xi(t),
$$

where

$$
\begin{aligned}
\Psi= & {\left[\begin{array}{ccccc}
\Gamma_{11} & 0 & -P C(t) & P A(t)+W_{1} K_{1} & P B(t) \\
* & \Gamma_{22} & 0 & 0 & W_{2} K_{1} \\
* & * & -\beta P & 0 & 0 \\
* & * & * & -W_{1} & 0 \\
* & * & * & * & -W_{2}
\end{array}\right] } \\
& +\left[\begin{array}{c}
H_{1}^{T}(t) P \\
H_{2}^{T}(t) P \\
H_{3}^{T}(t) P \\
0 \\
0
\end{array}\right] P^{-1}\left[\begin{array}{c}
H_{1}^{T}(t) P \\
H_{2}^{T}(t) P \\
H_{3}^{T}(t) P \\
0 \\
0
\end{array}\right], \\
\Gamma_{11}= & 2 \eta P+\alpha q P+\beta q P-W_{1} K_{2}-\lambda P, \\
\Gamma_{22}= & -\alpha P-W_{2} K_{2} .
\end{aligned}
$$

Using the similar method for uncertain parameters as above and from (10), we can get $\Psi<0$. Then we have $\mathbb{E} W(t)<0$; that is,

$$
\begin{aligned}
& \mathbb{E} \mathscr{L} V(t)+\alpha(q \mathbb{E} V(t)-\mathbb{E} V(t-\tau(t))) \\
& +\beta(q \mathbb{E} V(t)-\mathbb{E} V(t-\sigma(t)))<\lambda \mathbb{E} V(t),
\end{aligned}
$$

$$
t \neq t_{k} \text {. }
$$

Let $\lambda_{1}=\lambda_{\max }(P), \lambda_{0}=\lambda_{\min }(P)$. For any $\varepsilon>0$, there exists a $\delta>0$, such that $q \lambda_{1} \delta^{2}<\lambda_{0} \varepsilon^{2}$.

In the following, we will prove that when the initial function $\phi$ satisfies $\mathbb{E}\|\phi\|<\delta$, we have

$$
\mathbb{E} V(t)<\lambda_{0} \varepsilon^{2}, \quad t \geq t_{0}-\rho .
$$


First, for $t \in\left[t_{0}-\rho, t_{0}\right]$,

$$
\begin{aligned}
\mathbb{E} V(t) & =\mathbb{E}\left(e^{2 \eta t} y^{T}(t) P y(t)\right) \\
& \leq \lambda_{1} \mathbb{E}\|\phi\|^{2} \leq \lambda_{1} \delta^{2}<\frac{1}{q} \lambda_{0} \varepsilon^{2}<\lambda_{0} \varepsilon^{2} .
\end{aligned}
$$

Then we will prove that

$$
\mathbb{E} V(t)<\lambda_{0} \varepsilon^{2}, \quad t \in\left[t_{0}, t_{1}\right) .
$$

If the above inequality does not hold, then there exist $t^{*}=$ $\inf \left\{t \in\left(t_{0}, t_{1}\right): \mathbb{E} V(t) \geq \lambda_{0} \varepsilon^{2}\right\}$, such that $\mathbb{E} V\left(t^{*}\right)=\lambda_{0} \varepsilon^{2}$. Set $\bar{t}=\sup \left\{t \in\left[t_{0}, t^{*}\right): \mathbb{E} V(t) \leq(1 / q) \lambda_{0} \varepsilon^{2}\right\}$; then $\bar{t} \in\left(t_{0}, t^{*}\right)$ and $\mathbb{E} V(\bar{t})=(1 / q) \lambda_{0} \varepsilon^{2}$. Hence for all $t \in\left(\bar{t}, t^{*}\right), q \mathbb{E} V(t) \geq \lambda_{0} \varepsilon^{2}>$ $\mathbb{E} V(t+\theta), \forall \theta \in[-\rho, 0]$, which implies that

$$
\begin{array}{r}
q \mathbb{E} V(t)>\mathbb{E} V(t-\tau(t)), \quad q \mathbb{E} V(t)>\mathbb{E} V(t-\sigma(t)) \\
\text { for } t \in\left(\bar{t}, t^{*}\right) .
\end{array}
$$

It follows from (20) that for any $\alpha>0, \beta>0$ and $t \in\left(\bar{t}, t^{*}\right)$, we have

$$
\begin{aligned}
D^{+} \mathbb{E} V(t)= & \mathbb{E} \mathscr{L} V(t)<\mathbb{E} \mathscr{L} V(t) \\
& +\alpha[q \mathbb{E} V(t)-\mathbb{E} V(t-\tau(t))] \\
& +\beta[q \mathbb{E} V(t)-\mathbb{E} V(t-\sigma(t))]<\lambda \mathbb{E} V(t),
\end{aligned}
$$

which leads to $\mathbb{E} V\left(t^{*}\right) \leq \mathbb{E} V(\bar{t}) e^{\lambda\left(t^{*}-\bar{t}\right)} \leq \mathbb{E} V(\bar{t}) e^{\lambda \Delta_{\max }}=$ $(1 / q) e^{\lambda \Delta_{\max }} \lambda_{0} \varepsilon^{2}<\lambda_{0} \varepsilon^{2}$. This is a contradiction.

Thus (23) holds.

Now we assume that for some $m \in \mathbb{N}, \mathbb{E} V(t)<\lambda_{0} \varepsilon^{2}, t \in$ $\left[t_{0}-\rho, t_{m}\right)$; we will prove that

$$
\mathbb{E} V(t)<\lambda_{0} \varepsilon^{2}, \quad t \in\left[t_{m}, t_{m+1}\right)
$$

From (13), we have

$$
\begin{aligned}
\mathbb{E} V\left(t_{m}\right) \leq & \mu_{1} \mathbb{E} V\left(t_{k}^{-}\right)+\mu_{2} e^{2 \eta \tau} \mathbb{E} V\left(t_{k}^{-}-\tau\left(t_{k}\right)\right) \\
& +\mu_{3} e^{2 \eta \sigma} \mathbb{E} V\left(t_{k}^{-}-\sigma\left(t_{k}\right)\right) \\
< & \left(\mu_{1}+\mu_{2} e^{2 \eta \tau}+\mu_{3} e^{2 \eta \sigma}\right) \lambda_{0} \varepsilon^{2} \\
= & \frac{1}{q} \lambda_{0} \varepsilon^{2}<\lambda_{0} \varepsilon^{2} .
\end{aligned}
$$

Suppose (26) does not hold; then there exists $t^{*}=\inf \{t \in$ $\left.\left(t_{m}, t_{m+1}\right): \mathbb{E} V(t) \geq \lambda_{0} \varepsilon^{2}\right\}$ and $\mathbb{E} V\left(t^{*}\right)=\lambda_{0} \varepsilon^{2}$. Set $\bar{t}=\sup \{t \in$ $\left.\left[t_{m}, t^{*}\right): \mathbb{E} V(t) \leq(1 / q) \lambda_{0} \varepsilon^{2}\right\}$, and then from (27), $\bar{t} \in\left(t_{m}, t^{*}\right)$ and $\mathbb{E} V(\bar{t})=(1 / q) \lambda_{0} \varepsilon^{2}$. In the sequel, the proof is very similar with the proof of (23). Therefore (26) holds. By mathematical induction, inequality (21) holds. This together with $\mathbb{E} V(t) \geq$ $\lambda_{0} e^{2 \eta t} \mathbb{E}|y(t)|^{2}$, we have

$$
\mathbb{E}|y(t)|<\varepsilon e^{-\eta t}
$$

This completes the proof of Theorem 4 .

Remark 5. Theorem 4 shows that robustly exponential stability of system (1) can be achieved by adjusting suitable impulsive control and appropriate impulsive intervals even if the given networks without impulses may be unstable or chaotic themselves.

Remark 6. In [5], the authors investigated the stability of neural networks with delayed leakage term and impulsive perturbations. However, the neural networks without impulses must be stable. Moreover, parameter uncertainties and stochastic effects were not taken into account in the models. Hence, the results in this paper have wider adaptive range.

Theorem 7. Suppose that assumptions $\left(A_{1}\right)-\left(A_{4}\right)$ hold and for prescribed scalars $\Delta_{\min }>0$, choose positive scalars $\alpha, \beta, \mu_{1}$, $\mu_{2}, \mu_{3}$, satisfying $\mu_{1}+\mu_{2}+\mu_{3} \geq 1$ and $\mu_{1}+\mu_{2} e^{c \tau}+\mu_{3} e^{c \sigma}<$ $e^{c \Delta_{\text {min }}}$. Then the equilibrium point of system (1) is robustly exponentially stable in the mean square over any impulse time sequences satisfying $\inf \left\{t_{k}-t_{k-1} \mid k=1,2, \ldots\right\} \geq \Delta_{\min }$, if there exist positive definite matrix $P$ and positive definite diagonal matrices $W_{1}, W_{2}$ and positive constants $\varepsilon_{1}, \varepsilon_{2}, \varepsilon_{3}$ such that (8) and the following LMI hold:

$$
\left[\begin{array}{cccccccc}
\Omega_{11} & \varepsilon_{3} N_{1}^{T} N_{2} & -P C+\varepsilon_{3} N_{1}^{T} N_{3} & P A+W_{1} K_{1} & P B & H_{1}^{T} P & P E_{1} & 0 \\
* & \Omega_{22} & \varepsilon_{3} N_{2}^{T} N_{3} & 0 & W_{2} K_{1} & H_{2}^{T} P & 0 & 0 \\
* & * & \Omega_{33} & -\varepsilon_{2} M_{1}^{T} M_{2} & -\varepsilon_{2} M_{1}^{T} M_{3} & H_{3}^{T} P & 0 & 0 \\
* & * & * & -W_{1}+\varepsilon_{2} M_{2}^{T} M_{2} & \varepsilon_{2} M_{2}^{T} M_{3} & 0 & 0 & 0 \\
* & * & * & * & -W_{2}+\varepsilon_{2} M_{3}^{T} M_{3} & 0 & 0 & 0 \\
* & * & * & * & * & -P & 0 & P E_{2} \\
* & * & * & * & * & * & -\varepsilon_{2} I & 0 \\
* & * & * & * & * & * & * & -\varepsilon_{3} I
\end{array}\right]<0,
$$


where $\Omega_{11}=\left[(\alpha+\beta)\left(\mu_{1}+\mu_{2} e^{c \tau}+\mu_{3} e^{c \sigma}\right)+\ln \left(\mu_{1}+\mu_{2} e^{c \tau}+\right.\right.$ $\left.\left.\mu_{3} e^{c \sigma}\right) / \Delta_{\min }\right] P-W_{1} K_{2}+\varepsilon_{3} N_{1}^{T} N_{1}$ and $\Omega_{22}=-\alpha P-W_{2} K_{2}+$ $\varepsilon_{3} N_{2}^{T} N_{2}, \Omega_{33}=-\beta P+\varepsilon_{2} M_{1}^{T} M_{1}+\varepsilon_{3} N_{3}^{T} N_{3}$.
Proof. Since the matrix inequality (29) holds, we can choose small enough scalars $\eta>0, h>0$ satisfying $\ln \left(\mu_{1}+\mu_{2} e^{c \tau}+\right.$ $\left.\mu_{3} e^{c \sigma}+2 h\right) / \Delta_{\text {min }}+2 \eta \leq c$, such that the following matrix inequality holds:

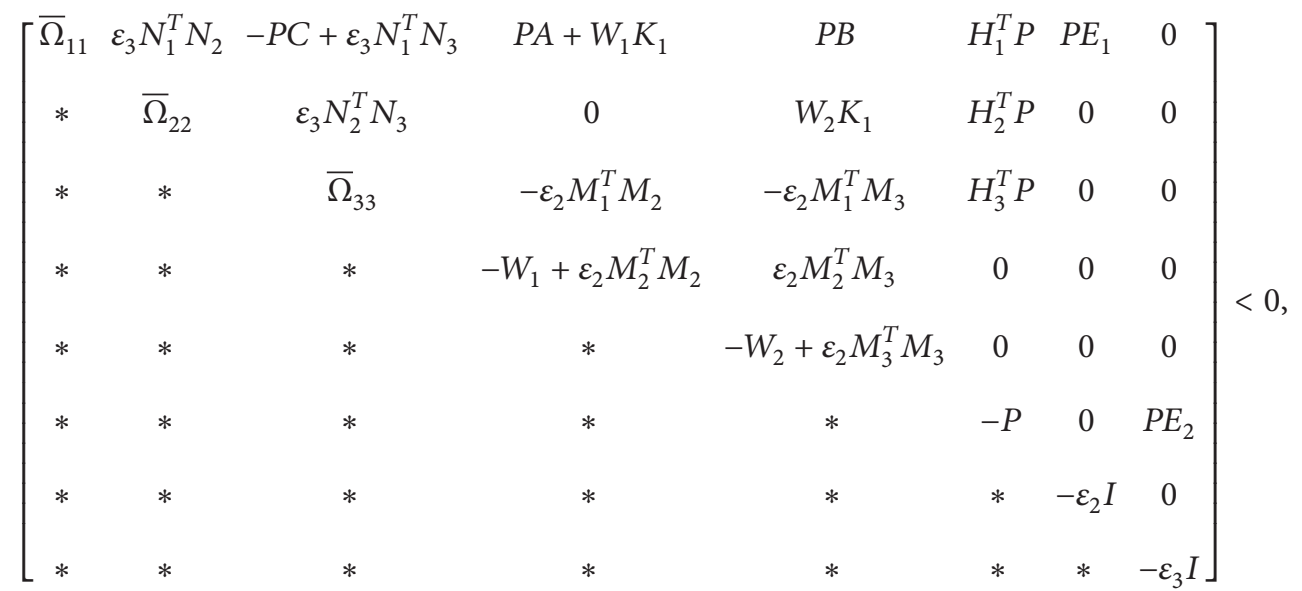

where $\bar{\Omega}_{11}=\left[2 \eta+(\alpha+\beta)\left(\mu_{1}+\mu_{2} e^{c \tau}+\mu_{3} e^{c \sigma}+h\right)+\ln \left(\mu_{1}+\right.\right.$ $\left.\left.\mu_{2} e^{c \tau}+\mu_{3} e^{c \sigma}+2 h\right) / \Delta_{\text {min }}\right] P-W_{1} K_{2}+\varepsilon_{3} N_{1}^{T} N_{1}, \bar{\Omega}_{22}=-\alpha e^{-2 \eta \tau} P-$ $W_{2} K_{2}+\varepsilon_{3} N_{2}^{T} N_{2}$, and $\bar{\Omega}_{33}=-\beta e^{-2 \eta \sigma} P+\varepsilon_{2} M_{1}^{T} M_{1}+\varepsilon_{3} N_{3}^{T} N_{3}$.

Set $q=\mu_{1}+\mu_{2} e^{c \tau}+\mu_{3} e^{c \sigma}+h, \lambda=\ln \left(\mu_{1}+\mu_{2} e^{c \tau}+\mu_{3} e^{c \sigma}+\right.$ 2h) $/ \Delta_{\text {min }}$; then $\lambda>0, q>1, e^{\lambda \Delta_{\min }}>q$.

Define Lyapunov function

$$
V(t, y(t))=e^{2 \eta t} y^{T}(t) P y(t)
$$

and function

$$
\begin{aligned}
W(t)= & \mathscr{L} V(t)+\alpha(q V(t)-V(t-\tau(t))) \\
& +\beta(q V(t)-V(t-\sigma(t)))+\lambda V(t) .
\end{aligned}
$$

Similar to the proof Theorem 4, if (8) and (29) hold, we have

$$
\begin{aligned}
V\left(t_{k}\right)= & e^{2 \eta t} y^{T}\left(t_{k}\right) P y\left(t_{k}\right) \\
\leq & \mu_{1} V\left(t_{k}^{-}\right)+\mu_{2} e^{2 \eta \tau} V\left(t_{k}^{-}-\tau\left(t_{k}\right)\right) \\
& +\mu_{3} e^{2 \eta \sigma} V\left(t_{k}^{-}-\sigma\left(t_{k}\right)\right), \quad t=t_{k},
\end{aligned}
$$

and $\mathbb{E} W(t)<0$ for $t \neq t_{k}$, which implies that

$$
\begin{aligned}
& \mathbb{E} \mathscr{L} V(t)+\alpha(q \mathbb{E} V(t)-\mathbb{E} V(t-\tau(t))) \\
& \quad+\beta(q \mathbb{E} V(t)-\mathbb{E} V(t-\sigma(t)))<-\lambda \mathbb{E} V(t) .
\end{aligned}
$$

For any $\varepsilon>0$, there exists $\delta>0$, such that $q \lambda_{1} \delta^{2}<\lambda_{0} \varepsilon^{2}$. In the following, we will prove that when the initial function $\phi \in P C_{\mathscr{F}_{0}}^{b}\left([-\rho, 0] ; \mathbb{R}^{n}\right)$ satisfies $\mathbb{E}\|\phi\|<\delta$, we have

$$
\mathbb{E} V(t)<\lambda_{0} \varepsilon^{2}, \quad t \geq t_{0}-\rho .
$$

First, for $t \in\left[t_{0}-\rho, t_{0}\right]$,

$$
\begin{aligned}
\mathbb{E} V(t) & =\mathbb{E} e^{2 \eta t} y^{T}(t) P y(t) \leq \lambda_{1} \mathbb{E}\|\phi\|^{2} \leq \lambda_{1} \delta^{2} \\
& <\frac{1}{q} \lambda_{0} \varepsilon^{2}<\lambda_{0} \varepsilon^{2} .
\end{aligned}
$$

Then we will prove that

$$
\mathbb{E} V(t)<\lambda_{0} \varepsilon^{2}, \quad t \in\left[t_{0}, t_{1}\right)
$$

If (37) does not hold, there exists $t^{*}=\inf \left\{t \in\left[t_{0}, t_{1}\right)\right.$ : $\left.\mathbb{E} V(t) \geq \lambda_{0} \varepsilon^{2}\right\}$. From (36), $t^{*} \in\left(t_{0}, t_{1}\right)$ and $\mathbb{E} V\left(t^{*}\right)=\lambda_{0} \varepsilon^{2}$. Set $\bar{t}=\sup \left\{t \in\left[t_{0}, t^{*}\right): \mathbb{E} V(t) \leq(1 / q) \lambda_{0} \varepsilon^{2}\right\}$; then $\bar{t} \in\left(t_{0}, t^{*}\right)$ and $\mathbb{E} V(\bar{t})=(1 / q) \lambda_{0} \varepsilon^{2}$.

So for $t \in\left(\bar{t}, t^{*}\right)$ and any $\theta \in[-\rho, 0]$, we have $q \mathbb{E} V(t) \geq$ $\lambda_{0} \varepsilon^{2}>\mathbb{E} V(t+\theta)$. It follows from (34) that for any $\alpha>0$, $\beta>0$ and $t \in\left(\bar{t}, t^{*}\right)$,

$$
\begin{aligned}
D^{+} \mathbb{E} V(t)= & \mathbb{E} \mathscr{L} V(t) \leq \mathbb{E} \mathscr{L} V(t) \\
& +\alpha[q \mathbb{E} V(t)-\mathbb{E} V(t-\tau(t))] \\
& +\beta[q \mathbb{E} V(t)-\mathbb{E} V(t-\sigma(t))]<-\lambda \mathbb{E} V(t) .
\end{aligned}
$$

Then we have $\lambda_{0} \varepsilon^{2}=\mathbb{E} V\left(t^{*}\right)<\mathbb{E} V(\bar{t}) e^{-\lambda\left(t^{*}-\bar{t}\right)} \leq \mathbb{E} V(\bar{t})=$ $(1 / q) \lambda_{0} \varepsilon^{2}<\lambda_{0} \varepsilon^{2}$. This is a contradiction. Thus (37) holds.

Suppose that for some $m \in \mathbb{N}, \mathbb{E} V(t)<\lambda_{0} \varepsilon^{2}, t \in\left[t_{0}-\right.$ $\left.\rho, t_{m}\right)$. We will prove that

$$
\mathbb{E} V(t)<\lambda_{0} \varepsilon^{2}, \quad t \in\left[t_{m}, t_{m+1}\right) .
$$

We first claim that $\mathbb{E} V\left(t_{m}^{-}\right) \leq(1 / q) \lambda_{0} \varepsilon^{2}$. The proof is very similar to $[18,19]$, so we omit it here. Next, we show that

$$
\mathbb{E} V\left(t_{m}^{-}-\sigma\left(t_{m}\right)\right) \leq \frac{1}{q} e^{\lambda \sigma} \lambda_{0} \varepsilon^{2} .
$$


Suppose not; then we have $\mathbb{E} V\left(t_{m}^{-}-\sigma\left(t_{m}\right)\right)>(1 / q) e^{\lambda \sigma} \lambda_{0} \varepsilon^{2}$. Without loss of generality, we assume $t_{m}-\sigma\left(t_{m}\right) \in\left(t_{l-1}, t_{l}\right], l \in$ $\mathbb{N}, l \leq m$. There are two cases to be considered.

Case 1. $\mathbb{E} V(t)>(1 / q) e^{\lambda \sigma} \lambda_{0} \varepsilon^{2}$ for any $t \in\left[t_{l-1}, t_{m}-\sigma\left(t_{m}\right)\right)$. Then we have

$$
\begin{gathered}
q \mathbb{E} V(t)>e^{\lambda \sigma} \lambda_{0} \varepsilon^{2} \geq \lambda_{0} \varepsilon^{2}>\mathbb{E} V(t+\theta), \\
\forall \theta \in[-\rho, 0], \quad t \in\left[t_{l-1}, t_{m}-\sigma\left(t_{m}\right)\right) .
\end{gathered}
$$

It follows from (34) that for any $\alpha>0, \beta>0$ and $t \in\left(t_{l-1}, t_{m}-\right.$ $\left.\sigma\left(t_{m}\right)\right)$ (38) holds, which leads to

$$
\begin{aligned}
\mathbb{E} V\left(t_{m}^{-}-\sigma\left(t_{m}\right)\right) & <\mathbb{E} V\left(t_{l-1}\right) e^{-\lambda\left(t_{m}-\sigma\left(t_{m}\right)-t_{l-1}\right)} \\
& <e^{-\lambda \Delta_{\min }} e^{\lambda \sigma} \lambda_{0} \varepsilon^{2}<\frac{1}{q} e^{\lambda \sigma} \lambda_{0} \varepsilon^{2} .
\end{aligned}
$$

This is a contradiction.

Case 2. There exist some $t \in\left[t_{l-1}, t_{m}-\sigma\left(t_{m}\right)\right)$, such that $\mathbb{E} V(t) \leq(1 / q) e^{\lambda \sigma} \lambda_{0} \varepsilon^{2}$

Set $\bar{t}=\sup \left\{t \in\left[t_{l-1}, t_{m}-\sigma\left(t_{m}\right)\right): \mathbb{E} V(t) \leq(1 / q) e^{\lambda \sigma} \lambda_{0} \varepsilon^{2}\right\}$. Then we have $\bar{t} \in\left[t_{l-1}, t_{m}^{-}-\sigma\left(t_{m}\right)\right)$ and $\mathbb{E} V(\bar{t})=(1 / q) e^{\lambda \sigma} \lambda_{0} \varepsilon^{2}$. Hence for $t \in\left(\bar{t}, t_{m}-\sigma\left(t_{m}\right)\right)$, we have

$$
q \mathbb{E} V(t) \geq e^{\lambda \sigma} \lambda_{0} \varepsilon^{2} \geq \lambda_{0} \varepsilon^{2}>\mathbb{E} V(t+\theta), \quad \forall \theta \in[-\rho, 0] .
$$

It follows from (34) that for any $\alpha>0, \beta>0$ and $t \in\left(\bar{t}, t_{m}-\right.$ $\left.\sigma\left(t_{m}\right)\right)(38)$ holds, which leads to

$$
\begin{aligned}
\mathbb{E} V\left(t_{m}^{-}-\sigma\left(t_{m}\right)\right) & <\mathbb{E} V(\bar{t}) e^{-\lambda\left(t_{m}-\sigma\left(t_{m}\right)-\bar{t}\right)} \leq \mathbb{E} V(\bar{t}) \\
& =\frac{1}{q} e^{\lambda \sigma} \lambda_{0} \varepsilon^{2}
\end{aligned}
$$

This is a contradiction.

Therefore (40) holds. By the same methods, we can prove $\mathbb{E} V\left(t_{m}^{-}-\tau\left(t_{m}\right)\right) \leq(1 / q) e^{\lambda \tau} \lambda_{0} \varepsilon^{2}$. Then from (33), we get

$$
\begin{aligned}
\mathbb{E} V\left(t_{m}\right) \leq & \mu_{1} \mathbb{E} V\left(t_{m}^{-}\right)+\mu_{2} e^{2 \eta \tau} \mathbb{E} V\left(t_{m}^{-}-\tau\left(t_{m}\right)\right) \\
& +\mu_{3} e^{2 \eta \sigma} \mathbb{E} V\left(t_{m}^{-}-\sigma\left(t_{m}\right)\right) \\
\leq & \left(\mu_{1}+\mu_{2} e^{(2 \eta+\lambda) \tau}+\mu_{3} e^{(2 \eta+\lambda) \sigma}\right) \frac{1}{q} \lambda_{0} \varepsilon^{2} \\
\leq & \left(\mu_{1}+\mu_{2} e^{c \tau}+\mu_{3} e^{c \sigma}\right) \frac{1}{q} \lambda_{0} \varepsilon^{2}<\lambda_{0} \varepsilon^{2} .
\end{aligned}
$$

Suppose (39) does not hold; then there exist $t^{*}=\inf \{t \in$ $\left.\left(t_{m}, t_{m+1}\right): \mathbb{E} V(t) \geq \lambda_{0} \varepsilon^{2}\right\}$ and $\mathbb{E} V\left(t^{*}\right)=\lambda_{0} \varepsilon^{2}$. If $\mathbb{E} V(t)>$ $(1 / q) \lambda_{0} \varepsilon^{2}$ for all $t \in\left[t_{m}, t^{*}\right)$, set $\bar{t}=t_{m}$. Otherwise let $\bar{t}=$ $\sup \left\{t \in\left[t_{m}, t^{*}\right): \mathbb{E} V(t) \leq(1 / q) \lambda_{0} \varepsilon^{2}\right\}$. Then for $t \in\left(\bar{t}, t^{*}\right)$ and any $\theta \in[-\rho, 0]$, we have $q \mathbb{E} V(t) \geq \lambda_{0} \varepsilon^{2}>\mathbb{E} V(t+\theta)$. It follows from (34) that for any $\alpha>0, \beta>0$, and $t \in\left(\bar{t}, t^{*}\right)$, (38) holds. Then, $\lambda_{0} \varepsilon^{2}=\mathbb{E} V\left(t^{*}\right)<\mathbb{E} V(\bar{t}) e^{-\lambda\left(t^{*}-\bar{t}\right)} \leq \mathbb{E} V(\bar{t})=$ $(1 / q) \lambda_{0} \varepsilon^{2}<\lambda_{0} \varepsilon^{2}$.

This is a contradiction. Thus (39) holds.
The next proof is very similar to Theorem 4 . This completes the proof.

Remark 8. Theorem 7 shows that the system will remain exponentially stable on the condition that the impulses, which may destabilize the system, do not occur too frequently.

Remark 9. If there is no leakage delay, that is, $\sigma(t)=0$, $H_{3}(t)=0, G_{3 k}(t)=0, k \in \mathbb{N}, t \geq 0$, then the system (4) is the one investigated in [19]. If there is no stochastic perturbation either, then system (4) is the one investigated in $[18,20]$.

\section{Examples}

In this section, we present some examples to verify the effectiveness of the theoretical results.

Example 1. Consider (4) with two neurons. The uncertain parameters satisfy assumption $\left(\mathrm{A}_{4}\right)$, where $C=\left[\begin{array}{ll}7 & 0 \\ 0 & 6\end{array}\right], A=$ $\left[\begin{array}{cc}0.6 & -0.2 \\ 0.7 & 0.5\end{array}\right], B=\left[\begin{array}{cc}0.5 & -0.1 \\ -1.2 & -0.9\end{array}\right], H_{1}=H_{2}=H_{3}=0.03 I, M_{1}=M_{2}=$ $M_{3}=0.03 I, G_{1 k}=G_{2 k}=G_{3 k}=0.1 I, k \in \mathbb{N}, N_{1}=N_{2}=$ $N_{3}=0.3 I, U_{1}=U_{2}=U_{3}=0.1 I, E_{1}=E_{2}=E_{3}=0.03 I$. $\tau<+\infty, \sigma<+\infty . g_{1}(y)=g_{2}(y)=|y+1|-|y-1|$, and it is obvious that $K_{1}=I, K_{2}=0$. Choose $\mu_{1}=0.05, \mu_{2}=0.1, \mu_{3}=$ $0.1, \alpha=\beta=0.5$, for $t_{k}-t_{k-1} \leq \Delta_{\max }=0.01$; then the LMIs in Theorem 4 have the following feasible solution via MATLAB LMI toolbox: $\varepsilon_{1}=1.2054, \varepsilon_{2}=11.5508, \varepsilon_{3}=0.7167$, $P=\left[\begin{array}{ll}1.2516 & 0.0492 \\ 0.0492 & 1.1285\end{array}\right], W_{1}=\left[\begin{array}{cc}9.7101 & 0 \\ 0 & 10.6592\end{array}\right], W_{2}=\left[\begin{array}{cc}0.3534 & 0 \\ 0 & 0.2846\end{array}\right]$. Thus from Theorem 4 , the equilibrium $(0,0)^{T}$ of system $(4)$ is robustly exponentially stable in the mean square.

Example 2. We consider neural network shown in [5] as follows:

$$
\dot{x}(t)=\left[\begin{array}{cc}
-9 & 0 \\
0 & -9
\end{array}\right] x(t-\sigma)+\left[\begin{array}{cc}
1 & 1 \\
-1 & 1
\end{array}\right] f(x(t)), \quad t>0,
$$

where $f_{1}(s)=f_{2}(s)=\tanh (s)$. As shown in [5], the system (46) is stable when $\sigma=0$, and it becomes unstable when $\sigma=$ 0.2 . We consider that the system has the following impulsive perturbation at times $t_{k}$ :

$$
\Delta x(t)=\left(G_{1 k}(t)-I\right) x\left(t^{-}\right), \quad t=t_{k}, k \in \mathbb{N},
$$

where $G_{1 k}=0.3 I, k \in \mathbb{N}$. It is obvious that $K_{1}=0.5 I, K_{2}=0$. For $t_{k}-t_{k-1} \leq \Delta_{\max }=0.01$, choosing $\mu_{1}=0.1, \mu_{2}=0.01$, $\mu_{3}=0.05, \alpha=0.5, \beta=0.5$, by using the LMI toolbox in MATLAB, a feasible solution of Theorem 4 is

$$
\begin{aligned}
& P=\left[\begin{array}{cc}
0.0210 & 0 \\
0 & 0.0210
\end{array}\right], \quad W_{1}=\left[\begin{array}{cc}
0.6321 & 0 \\
0 & 0.6321
\end{array}\right], \\
& W_{2}=\left[\begin{array}{cc}
0.0289 & 0 \\
0 & 0.0289
\end{array}\right], \quad \varepsilon_{1}=\varepsilon_{2}=\varepsilon_{3}=0.6748 .
\end{aligned}
$$

Thus from Theorem 4 , the equilibrium $(0,0)^{T}$ of system (46) is robustly exponentially stable in the mean square. It can be seen that the impulses play an important role in inducing the stability of neural network in the leakage time delay. 


\section{Conclusion}

Robust exponential stability of stochastic neural networks with time-varying delay in the leakage term under impulsive perturbations is investigated. The leakage delay is time varying and the impulsive perturbations depend not only on the current state of neurons at impulse times but also on the state of neurons in its recent history. Based on Lyapunov functions and Razumikhin techniques, some new criteria are derived. Some examples have been given to demonstrate that, even though the corresponding delayed neural networks without impulses are unstable, impulses may compensate the deviating trend.

\section{Conflict of Interests}

The authors declare that there is no conflict of interests regarding the publication of this paper.

\section{Acknowledgments}

The authors wish to thank the referees for their suggestions and comments. This work is supported by the National Science Foundation of China (11171374) and the Key National Science Foundation of Shandong Province (ZR2011AZ001).

\section{References}

[1] H. Zhao, "Global exponential stability and periodicity of cellular neural networks with variable delays," Physics Letters A, vol. 336, no. 4-5, pp. 331-341, 2005.

[2] Y. Wang, C. Lu, G. Ji, and L. Wang, "Global exponential stability of high-order Hopfield-type neural networks with Stype distributed time delays," Communications in Nonlinear Science and Numerical Simulation, vol. 16, no. 8, pp. 3319-3325, 2011.

[3] L. Wang, R. Zhang, and Y. Wang, "Global exponential stability of reaction-diffusion cellular neural networks with Stype distributed time delays," Nonlinear Analysis: Real World Applications, vol. 10, no. 2, pp. 1101-1113, 2009.

[4] K. Gopalsamy, "Leakage delays in BAM," Journal of Mathematical Analysis and Applications, vol. 325, no. 2, pp. 1117-1132, 2007.

[5] X. Li, X. Fu, and P. Balasubramaniam, "Existence, uniqueness and stability analysis of recurrent neural networks with time delay in the leakage term under impulsive perturbations," Nonlinear Analysis. Real World Applications, vol. 11, no. 5, pp. 4092-4108, 2010.

[6] X. Li, R. Rakkiyappan, and P. Balasubramaniam, "Existence and global stability analysis of equilibrium of fuzzy cellular neural networks with time delay in the leakage term under impulsive perturbations," Journal of the Franklin Institute, vol. 348, no. 2, pp. 135-155, 2011.

[7] Y. Wang, C. Zheng, and E. Feng, "Stability analysis of mixed recurrent neural networks with time delay in the leakage term under impulsive perturbations," Neurocomputing, vol. 119, pp. 454-461, 2013.

[8] S. Long, Q. Song, X. Wang, and D. Li, "Stability analysis of fuzzy cellular neural networks with time delay in the leakage term and impulsive perturbations," Journal of the Franklin Institute, vol. 349, no. 7, pp. 2461-2479, 2012.
[9] X. Li and X. Fu, "Effect of leakage time-varying delay on stability of nonlinear differential systems," Journal of the Franklin Institute, vol. 350, no. 6, pp. 1335-1344, 2013.

[10] B. Liu, "Global exponential stability for BAM neural networks with time-varying delays in the leakage terms," Nonlinear Analysis: Real World Applications, vol. 14, no. 1, pp. 559-566, 2013.

[11] M. Park, O. Kwon, J. Park, S. Lee, and E. Cha, "Synchronization criteria for coupled stochastic neural networks with timevarying delays and leakage delay," Journal of the Franklin Institute, vol. 349, no. 5, pp. 1699-1720, 2012.

[12] Q. Gan and Y. Liang, "Synchronization of chaotic neural networks with time delay in the leakage term and parametric uncertainties based on sampled-data control," Journal of the Franklin Institute, vol. 349, no. 6, pp. 1955-1971, 2012.

[13] Z. Guan, J. Lam, and G. Chen, "On impulsive autoassociative neural networks," Neural Networks, vol. 13, no. 1, pp. 63-69, 2000.

[14] Z. Guan and G. Chen, "On delayed impulsive Hopfield neural networks," Neural Networks, vol. 12, no. 2, pp. 273-280, 1999.

[15] S. Haykin, Neural Networks, Prentice-Hall, Upper Saddle River, NJ, USA, 1994

[16] X. Liang and L. Wang, "Exponential stability for a class of stochastic reaction-diffusion Hopfield neural networks with delays," Journal of Applied Mathematics, vol. 2012, Article ID 693163, 12 pages, 2012.

[17] D. Li, X. Wang, and D. Xu, "Existence and global p-exponential stability of periodic solution for impulsive stochastic neural networks with delays," Nonlinear Analysis. Hybrid Systems, vol. 6, no. 3, pp. 847-858, 2012.

[18] W. Chen and W. X. Zheng, "Robust stability and $H_{\infty}$-control of uncertain impulsive systems with time-delay," Automatica, vol. 45, no. 1, pp. 109-117, 2009.

[19] P. Cheng, F. Deng, and Y. Peng, "Robust exponential stability and delayed-state-feedback stabilization of uncertain impulsive stochastic systems with time-varying delay," Communications in Nonlinear Science and Numerical Simulation, vol. 17, no. 12, pp. 4740-4752, 2012.

[20] Y. Zhang, "Robust exponential stability of uncertain impulsive neural networks with time-varying delays and delayed impulses," Neurocomputing, vol. 74, no. 17, pp. 3268-3276, 2011.

[21] S. Wu, K. Li, and T. Huang, "Global exponential stability of static neural networks with delay and impulses: discrete-time case," Communications in Nonlinear Science and Numerical Simulation, vol. 17, no. 10, pp. 3947-3960, 2012.

[22] Y. Wang, L. Xie, and C. E. de Souza, "Robust control of a class of uncertain nonlinear systems," Systems and Control Letters, vol. 19, no. 2, pp. 139-149, 1992.

[23] S. Boyd, L. El Ghaoui, E. Feron, and V. Balakrishnan, Linear Matrix Inequalities in System and Control Theory, Society for Industrial and Applied Mathematics, Philadelphia, Pa, USA, 1994. 


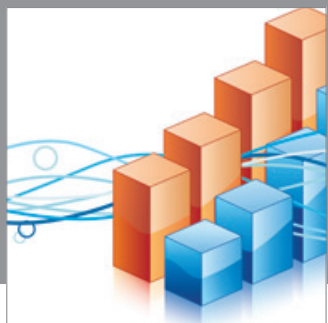

Advances in

Operations Research

mansans

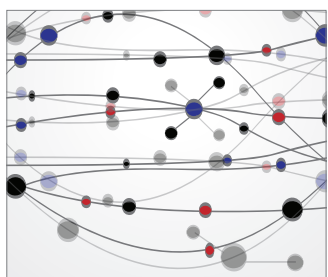

The Scientific World Journal
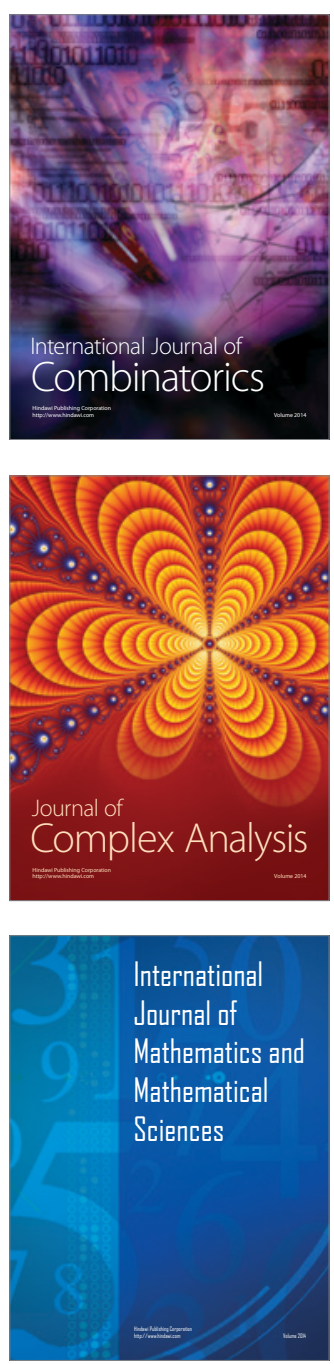
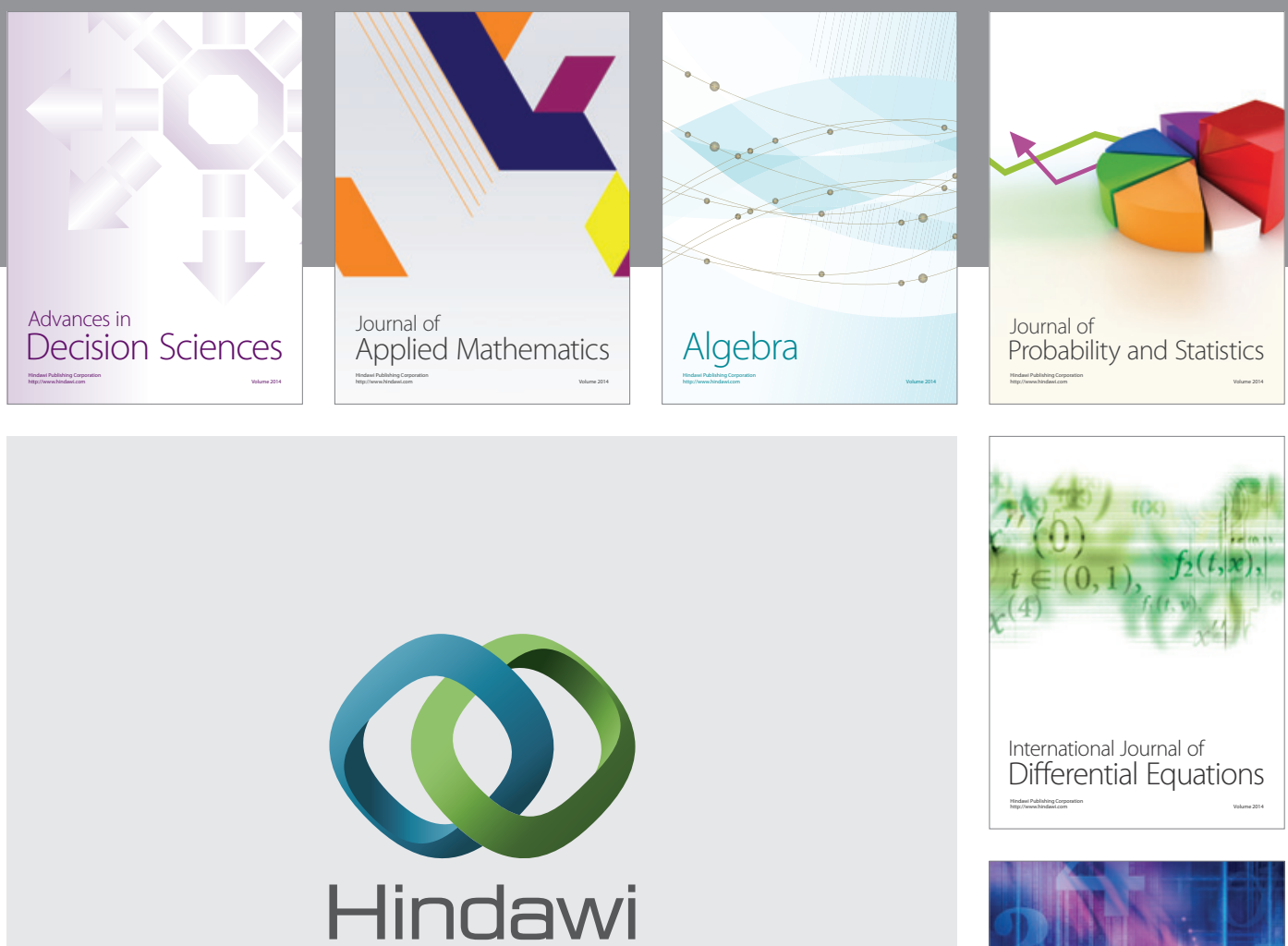

Submit your manuscripts at http://www.hindawi.com
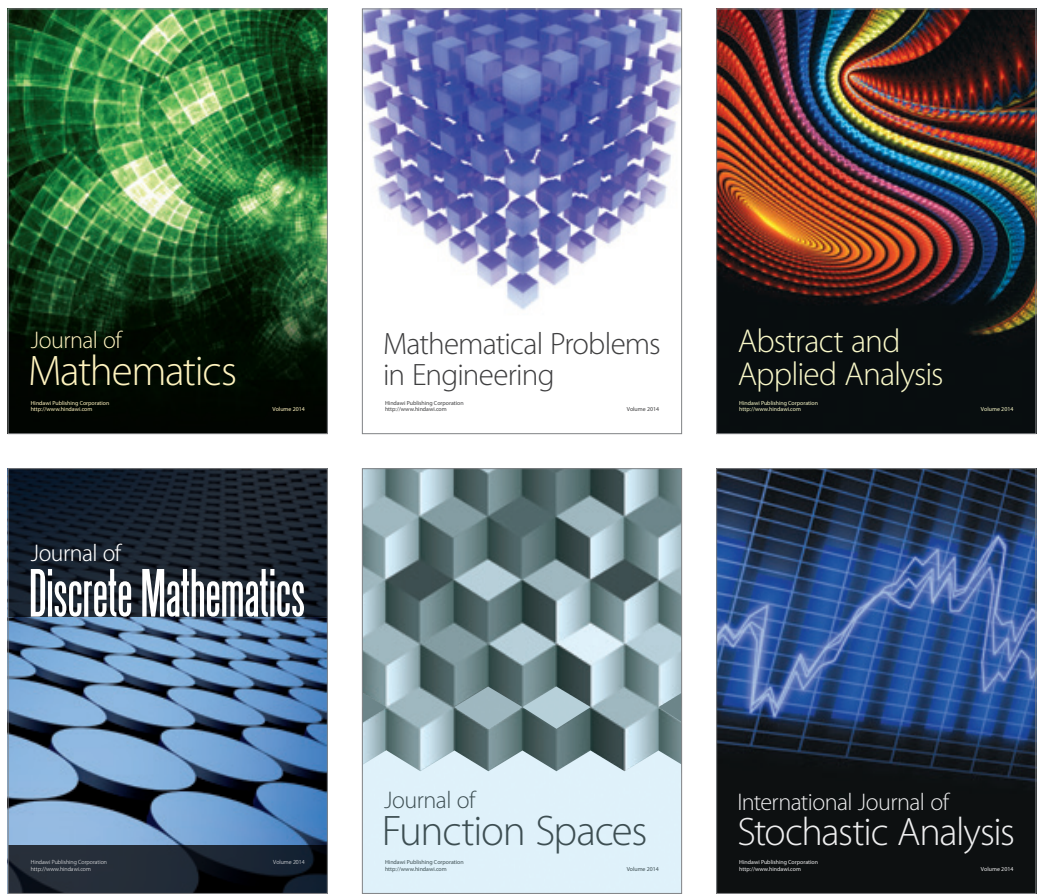

Journal of

Function Spaces

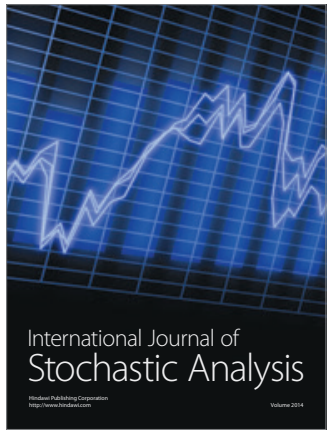

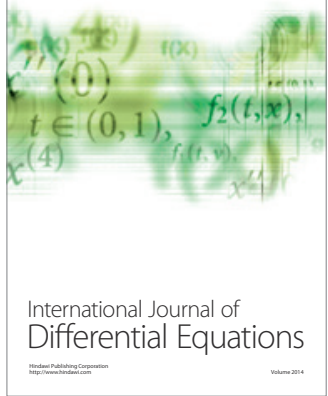
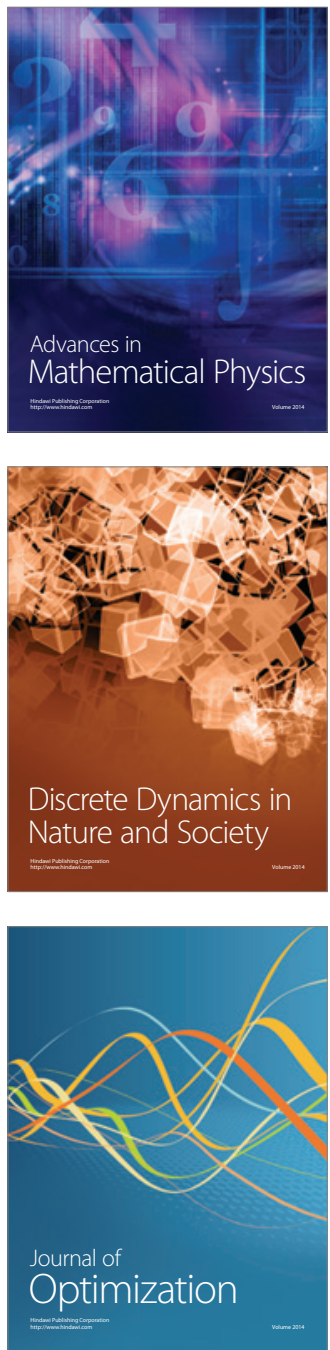